\title{
Retraction Note: Induction of a Pepper cDNA Encoding SAR8.2 Protein during the Resistance Response to Tobacco Mosaic Virus
}

\author{
Gil-je Lee, Ryoung Shin, Chang-Jin Park, Tae Hyoung Yoo, and Kyung-Hee Paek*
}

Retraction Note: Mol. Cells 12 (2001) 250-256

\begin{abstract}
Members of the editorial board have unanimously agreed to retract the article [Mol. Cells 12 (2001) 250-256] for potential misconducts mainly concerning manipulation and repeated uses of photomicrographs of control data internally along with mislabeling and/or externally in multiple publications. As specified in the "Instructions to Authors", Molecules and Cells (Mol. Cells) explicitly prohibits mis-representation or falsification of experimental data including duplication of previously published data. In the article, lanes 1-4 and lanes 5-7 of rRNA gels in Fig. 2B are identical to lanes 1-4 and lanes 10-12 in Fig. 3A, respectively; two EtBr gels of rRNA in Figs. 4A and 4B are identical, and rRNA gels in Figs. 5A, 5B, and $5 \mathrm{C}$ are identical as well.
\end{abstract}

